\title{
EVALUACIÓN MORFOMÉTRICA Y DIMORFISMO SEXUAL INTRA-POBLACIONAL DE Rhinoclemmys nasuta (Boulenger, 1902) EN UNA ZONA INSULAR CONTINENTAL DEL PACIFICO COLOMBIANO
}

\section{SEXUAL DIMORPHISM AND MORPHOMETRIC EVALUATION ON INTRA-POPULATION OF Rhinoclemmys nasuta (Boulenger, 1902) IN A CONTINENTAL INSULAR ZONE IN THE COLOMBIAN PACIFIC COAST}

\author{
PEREZ, JANETH VIVIANA. ${ }^{1,2^{*}}$ BIOL, ALEGRÍA, JORGE. BIOL. ${ }^{2^{*}}$ \\ ${ }^{1}$ Grupo de Investigación en Ecología Animal. Departamento de Biología. Universidad \\ del Valle. Cali. Colombia ${ }^{2}$ Fundacion Archelon. Cali. Colombia. \\ *Correspondencia: jviviana.perez@gmail.com, alegria.jluis@gmail.com.
}

\section{Resumen}

En el año 2006 se realizó un seguimiento de Rhinoclemmys nasuta en seis riachuelos de una zona insular continental del Pacífico colombiano (Isla Palma); con el propósito de evaluar la variación morfométrica intra-poblacional. Se capturaron 333 tortugas, y se realizó en cada individuo el registro de 10 medidas corporales: Largo del caparazón (LC SLM), Largo máximo del caparazón (LC max), Ancho del caparazón (AC m5-6), Ancho máximo del caparazón (AC max), Largo del plastrón (LP SLM), Largo máximo del plastron (LP max), Altura del caparazón (ALRC), la medida del puente (LP), Longitud de la cola pre-cloacal y pos-cloacal, con una media de (LC SLM) para hembras, machos y juveniles respectivamente de $(178,7 \mathrm{~mm}, 134,0 \mathrm{~mm}, 125,5 \mathrm{~mm})$. Se detectaron diferencias morfométricas significativas entre las hembras, machos y juveniles de $R$. nasuta en Isla Palma. Sin embargo, no se detectaron diferencias significativas entre los riachuelos estudiados. De las 10 variables morfológicas seleccionadas para el desarrollo de este estudio, la longitud pre-cloacal explicó el $84,23 \%$ de la variación entre hembras y machos, por lo que se propone el uso de esta medida como elemento apropiado para establecer el sexo de $R$. nasuta, descartando el uso de la longitud total de la cola para este fin.

Palabras clave: dimorfismo sexual, morfometria, tortugas, endemismo, Colombia. 


\begin{abstract}
In the year 2006, monitoring on Rhinoclemmys nasuta was carried out in six small streams in a continental insular zone on the Colombian Pacific coast (Palma Island) with the aim of evaluating the intrapopulational morphometric variation. Three hundred thirty-three tortoises were captured and 10 body measurements were recorded for each individual: length of the shell, maximum length of the shell, width of the shell, maximum width of the shell, length of the plastron, maximum length of the plastron, height of the shell, bridge length, pre-cloaca tail length and post-cloaca tail length with measures for females, males and young of $178.7 \mathrm{~mm}, 134.0 \mathrm{~mm}$ and $125.5 \mathrm{~mm}$, respectively. Significant morphometric differences were detected among $R$. nasuta female, male and young from Palma Island. Nevertheless, no significant differences were detected among the small streams studied. From the 10 morphological variables selected for the development of this study, the pre-cloacal tail length explained $84.23 \%$ of variation between females and males; accordingly the use of this measurement is proposed as appropriate for distinguishing the sex in $R$. nasuta, ruling out the total length of the tail for this purpose.
\end{abstract}

Key words: sexual dimorphism, morphometrics, tortoises, endemism, Colombia

\title{
Introducción
}

Las variaciones morfológicas llevadas al campo métrico, pueden representar cambios y divergencias en las arquitecturas óseas o dérmicas de los individuos. De tal manera que permiten contrastar las estructuras que representan estas divergencias, en el orden que sean medibles y ó cuantificables. Con base en esta aproximación, se identifican caracteres no redundantes que pueden estar asociados con diferentes aspectos generales de los individuos, especies o poblaciones, como hábitos, uso de hábitat, fisiología e incluso comportamiento reproductivo (TELLERÍA, 1991; ANÓNIMO, 2002).

Considerando la configuración anatómica de los individuos, se pueden identificar tres niveles de variación morfométrica que estarían fuertemente modulados por las condiciones físicas y químicas del entorno: entre géneros, entre edades y entre hábitats (TELLERIA, 1991). Esta categorización de la variabilidad morfométrica permite tener en consideración el funcionamiento de los mecanismos adaptativos, el rol de los individuos en la estructura social y su comportamiento, dado que la forma de los organismos no está dada solamente por su genética, sino también por su ecología y las presiones ambientales (SARA et al., 1999), difiriendo notablemente en su morfología dentro y entre las poblaciones (McCOY et al., 2006). Estas diferencias fenotípicas pueden cambiar incluso, el tipo y dirección de la evolución, la dinámica de la población, la 
determinación de los resultados en las interacciones de los sistemas ecológicos y la capacidad de adecuación a cambios ambientales (BOLKER et al., 2003; WERNER y PEACOR, 2003). Por lo tanto, es relevante evaluar las diferencias fenotípicas dentro y entre poblaciones ya que es una herramienta efectiva para establecer la respuesta individual y poblacional a presiones externas ya sean de origen natural 0 antropogénico.

En este sentido, el estudio sistémico de los caracteres morfométricos en las tortugas de agua dulce, ha permitido evaluar la persistencia de las características anatómicas, fisiológicas y mecánicas en este grupo. Al parecer, esta conformación morfológica les ha permitido enfrentar las condiciones adversas y variables del hábitat durante los últimos 200 millones de años (ARESCO y DOBIE, 2000), razón por la cual han colonizado variados ambientes como estanques, lagos, arroyos, grandes ríos, torrentes, estuarios, océanos, desiertos y bosques (ROMER, 1956).

La diversificación en la ocupación de hábitats por parte de las tortugas ha generado un alto grado de especialización especialmente en lo relacionado con patrones de locomoción y actividad fisiológica (DEPECKER et al., 2006). Además, estas adecuaciones y adaptaciones morfológicas generalmente están asociadas con mecanismos de defensa, facilidad en la obtención de alimentos, territorialidad, eventos de cortejo y copula, desplazamiento, anidación y termorregulación (WEATHERS y WHITE, 1971; SOLER y MARTÍNEZ, 2005; DEPECKER et al., 2006).

Uno de los principales retos en los procesos de conservación de las tortugas, ya sean terrestres, acuáticas o semiacuáticas, es la estimación fiable de parámetros demográficos. Sin embargo, estos estimados dependerán en gran medida con la exactitud en la que se puedan identificar los sexos de los individuos capturados (KELLER, 1997). Aunque el dimorfismo sexual de los adultos es frecuente entre las diferentes especies de tortugas, la identificación certera de los individuos juveniles es problemática, ya que no presentan caracteres sexuales evidentes lo que generalmente conduce a un error en la estimación de la proporción sexual de las poblaciones evaluadas (GIBBONS, 1990).

Rhinoclemmys nasuta Boulenger (1902), es una especie endémica del Chocó Biogeográfico clasificada como casi amenazada (NT) por la IUCN, de hábitos diurnos y nocturnos que habita en ríos, caños grandes o pequeños riachuelos, quebradas y esteros, y suele refugiarse en las orillas bajo las ramas, raicillas y troncos caídos (RUEDA et al., 2007). En cuanto a la historia natural de esta especie solo se cuenta 
con los registros de MEDEM (1962) y CORREDOR et al. (2007), siendo escasos los esfuerzos de conservación dirigida.

En el presente trabajo se realiza un análisis merístico de una población de $R$. nasuta en la localidad de Isla Palma (Bahía Málaga, Pacifico Colombiano). Teniendo en cuenta la variación probable interespecifica de las poblaciones, anidadas según los riachuelos en los que habitan, y las variaciones relacionadas con el ambiente en que se desarrollan, en conjunción con el estudio de los caracteres morfológicos medidos se permitirá la evaluación esquemática de los datos tomados de la población y la morfología de los individuos por localidad, evidenciando los caracteres inter poblacionales mediados según el ámbito de su desarrollo. Permitiendo con esto la adquisición de una herramienta merística para la identificación de individuos sub adultos o juveniles de la especie; y la diferenciación morfométrica de los individuos por poblaciones individualizadas entre riachuelos y por localidad o la isla en su totalidad.

\section{Materiales y Métodos}

Isla Palma está ubicada en Bahía Málaga ( $3^{\circ} 53^{\prime} \mathrm{N} ; 77^{\circ}{ }^{2} 1^{\prime} \mathrm{W}$ ), al Norte del Puerto de Buenaventura (Fig. 1); se encuentra rodeada de acantilados rocosos distribuidos uniformemente con alturas entre 6 y $15 \mathrm{~m}$, además posee playas arenosas que quedan descubiertas en bajamar. En este sector se presentan en el año dos periodos de alta pluviosidad en los meses de septiembre y noviembre y el otro con menor intensidad en los meses de abril y mayo; el promedio de precipitación anual es de $6000 \mathrm{~mm}$ y la humedad relativa es del 90\% (CANTERA et al., 1998). Estas características climáticas favorecen el desarrollo de abundantes salidas de agua dulce de la isla hacia el mar (AMAYA, 2003). La vegetación presente en la isla es típica de selva neotropical, predominando árboles entre 10 y $50 \mathrm{~m}$ de altura, arbusto de tallas pequeñas, helechos y diversidad de gramíneas (LOAIZA, 2006).

Para el desarrollo de esta investigación se realizaron seis campañas de muestreo durante los meses de marzo a noviembre del año 2006. En cada campaña, se realizaron monitoreos de Rhinoclemmys nasuta en 6 riachuelos de Isla Palma (Fig. 1), mediante inspección directa y captura manual. Todos los individuos capturados fueron identificados con una marca única siguiendo la metodología de CAGLE (1939) con ajustes en el sistema numérico de marcaje, generando muescas en las placas marginales del caparazón. 


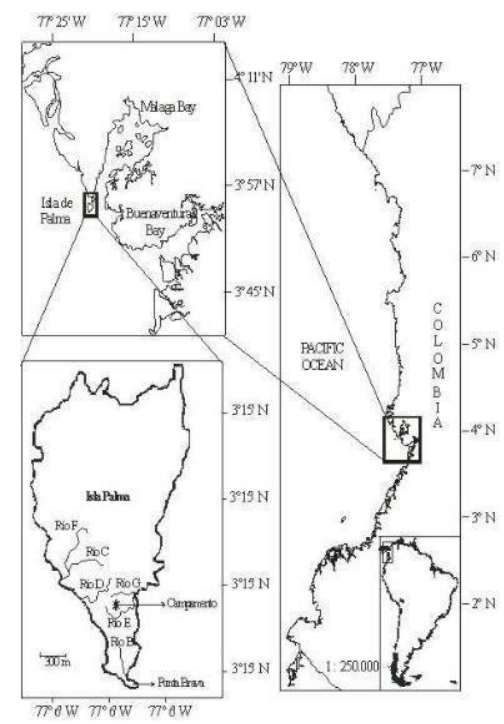

Figura 1. Ubicación geográfica del área de estudio Isla Palma, Pacífico Colombiano.

Se midieron con un calibrador 10 variables morfológicas, considerando lo propuesto por MEDEM (1976) y LOAIZA (2006) utilizando un calibrador metálico de precisión $0.01 \mathrm{~mm}$. Las variables seleccionadas fueron: Largo del caparazón (LCS); Largo máximo del caparazón (LC Max); Ancho del caparazón (AC m5-6), entre los escudos marginales cinco (5) y seis (6); Ancho máximo del caparazón (AC Max), se ubicó posterior a la medida estándar del ancho del caparazón; Largo del plastrón (LPS), está medida se realizó sobre la línea media del plastrón; Largo máximo del plastrón (LP Max), este registro se realizó desde los escudos gulares hasta los anales; Altura del caparazón (A. caparazón); Longitud del puente (L. puente) que une el plastrón y el caparazón; y la Longitud de la cola pre-cloacal y pos-cloacal. (Fig. 2).

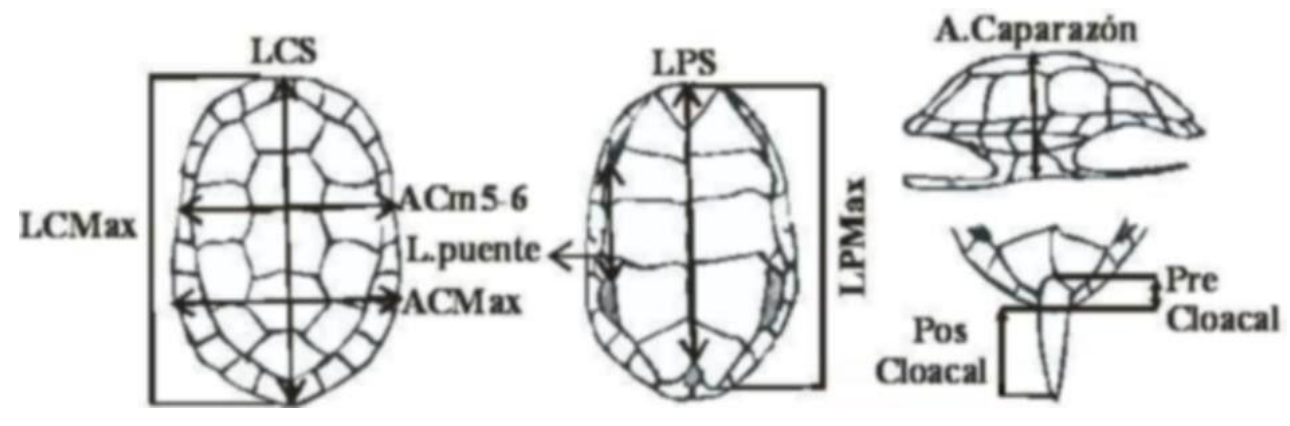

Figura 2. Medidas usadas en la determinación de la variación morfométrica.

Para definir el sexo de cada individuo se utilizaron criterios visuales como tamaño 
corporal, concavidad plastral y en algunos casos eversión de penes y palpación en la zona inguinal para detección de huevos en hembras. Se consideraron juveniles a los individuos que mediante criterios visuales y de tamaño no fueron posible sexar.

Con el propósito de identificar la variable morfométrica que más aporta a la variación merística y morfológica de las tortugas se utilizó un análisis de ordenamiento de componentes principales siguiendo a KELLER (1997), realizando un ajuste logarítmico preliminar para minimizar el efecto de la variabilidad en la talla y la relación alométrica entre medidas (ROWE, 1997; MERCHÁN, 2003). Adicionalmente se realizó un ANOVA factorial para identificar el grado de variabilidad de la estructura etaria y de las tortugas entre los diferentes riachuelos, evaluando el dimorfismo sexual en los adultos respecto a cada una de las variables morfométricas seleccionadas.

\section{Resultados}

La relación entre la longitud máxima del plastrón y el ancho máximo del caparazón de todos los individuos colectados sugiere la presencia de un fuerte dimorfismo sexual en R. nasuta (Fig. 3).

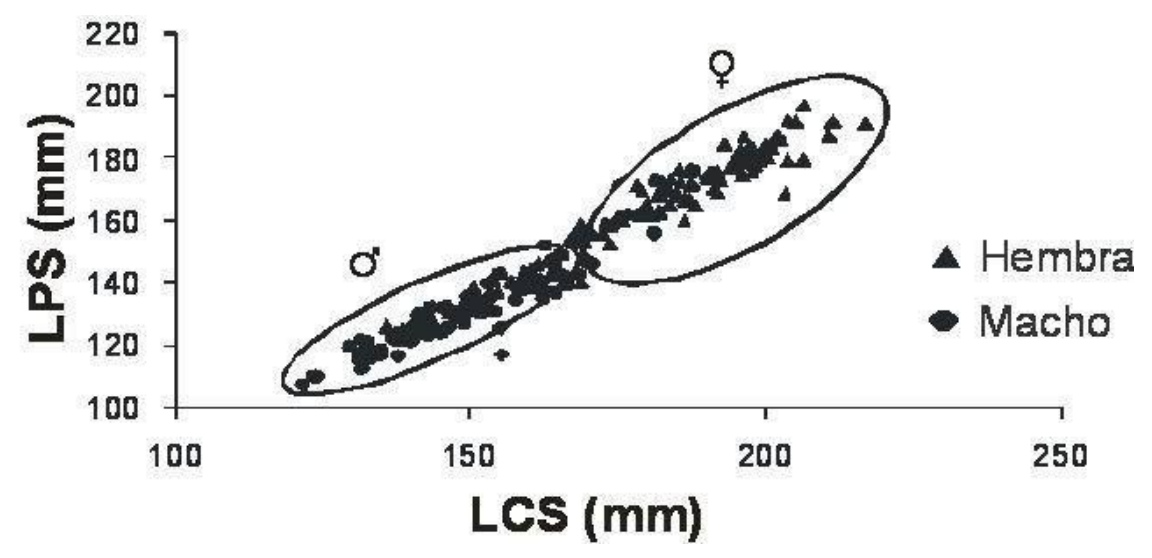

Figura 3. Relación entre la longitud del plastrón y del Caparazón entre hembras y machos.

En este sentido, con excepción de la longitud pos-cloacal, todas las medidas utilizadas para describir la Morfometría de la caparazón de $R$. nasuta fueron significativamente diferentes entre los machos y las hembras (Tabla 1).

Además, las variables que describen las longitudes máximas del caparazón (largo y ancho del caparazón y longitud del plastrón) fueron significativamente diferentes entre 
los sitios de colecta, siendo los machos del río espinoso significativamente más pequeños (Fig. 4).

Tabla 1. Análisis de Varianza de las medidas morfométricas, sexo y riachuelos.

\begin{tabular}{|c|c|c|c|c|}
\hline & \multicolumn{4}{|c|}{ Fuente } \\
\hline Variable & & Sexo & Rio & Sexo x Rio \\
\hline LCs & F & 32,3 & 1,00 & 1,20 \\
\hline & p & 0,00 & 0,44 & 0,32 \\
\hline LC max & F & 88,7 & 2,90 & 1,10 \\
\hline & p & 0,00 & 0,01 & 0,38 \\
\hline AC m5-6 & F & 92,0 & 1,40 & 0,30 \\
\hline & p & 0,00 & 0,23 & 0,90 \\
\hline AC max & F & 87,9 & 2,50 & 0,70 \\
\hline & p & 0,00 & 0,03 & 0,62 \\
\hline LPs & F & 89,5 & 2,00 & 0,80 \\
\hline & $\mathrm{p}$ & 0,00 & 0,09 & 0,52 \\
\hline LPmax & F & 91,4 & 2,90 & 0,80 \\
\hline & p & 0,00 & 0,01 & 0,58 \\
\hline Lpuente & F & 54,08 & 0,81 & 0,49 \\
\hline & p & 0,00 & 0,54 & 0,78 \\
\hline Alto Caparazón & F & 23,91 & 2,24 & 0,87 \\
\hline & p & 0,00 & 0,05 & 0,50 \\
\hline Pre-cloacal & F & 79,93 & 1,94 & 0,97 \\
\hline & p & 0,00 & 0,09 & 0,43 \\
\hline Pos-cloacal & F & 1,46 & 0,57 & 1,07 \\
\hline & p & 0,23 & 0,72 & 0,38 \\
\hline & & & & \\
\hline
\end{tabular}



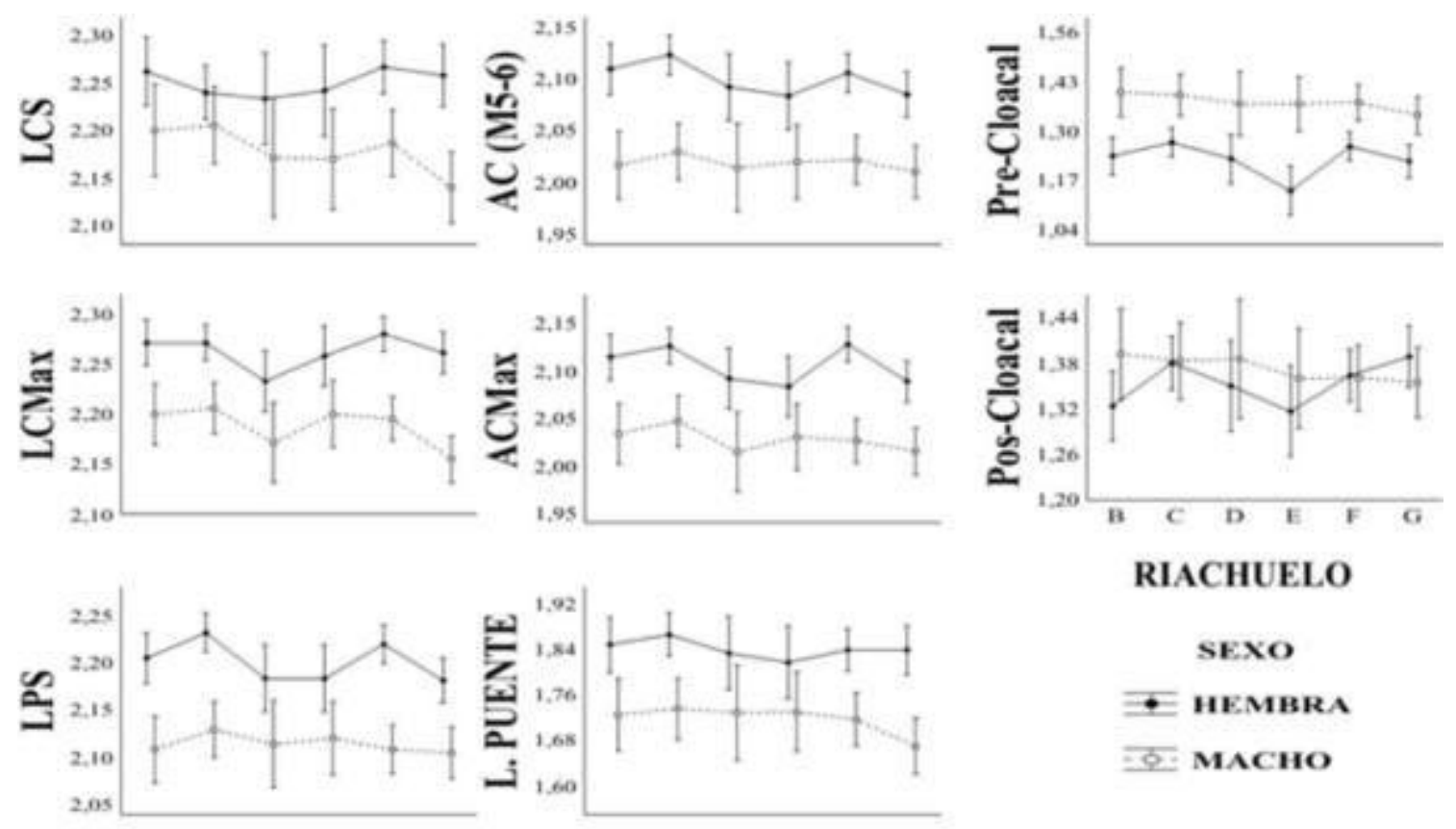

RIACHUELO
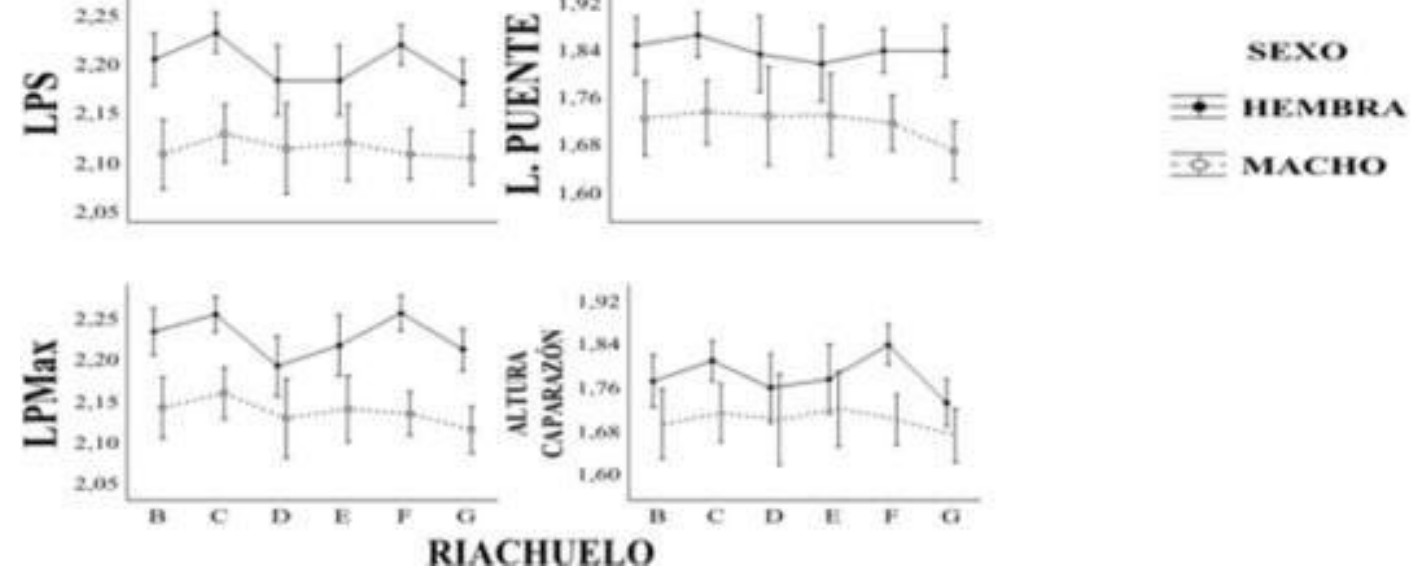

Figura 4. Gráfica descriptiva de las medidas morfométricas y su comparación entre riachuelos y sexo.

El análisis de componentes principales (ACP) no sugiere un ordenamiento definido espacialmente de la variación morfométrica para los individuos capturados en los diferentes riachuelos de Isla Palma (Fig. 5). Sin embargo, este análisis discriminó claramente la separación entre sexos, conformando tres agregaciones características de información (Fig. 6). Al evaluar el aporte el ACP considerando las 10 medidas morfométricas utilizadas se generó un factor que explicó el $84,3 \%$ de la variación merística total (Fig. 7), identificándose la longitud pre-cloacal como la variable morfométrica que discrimina en mayor medida entre machos y hembras. 


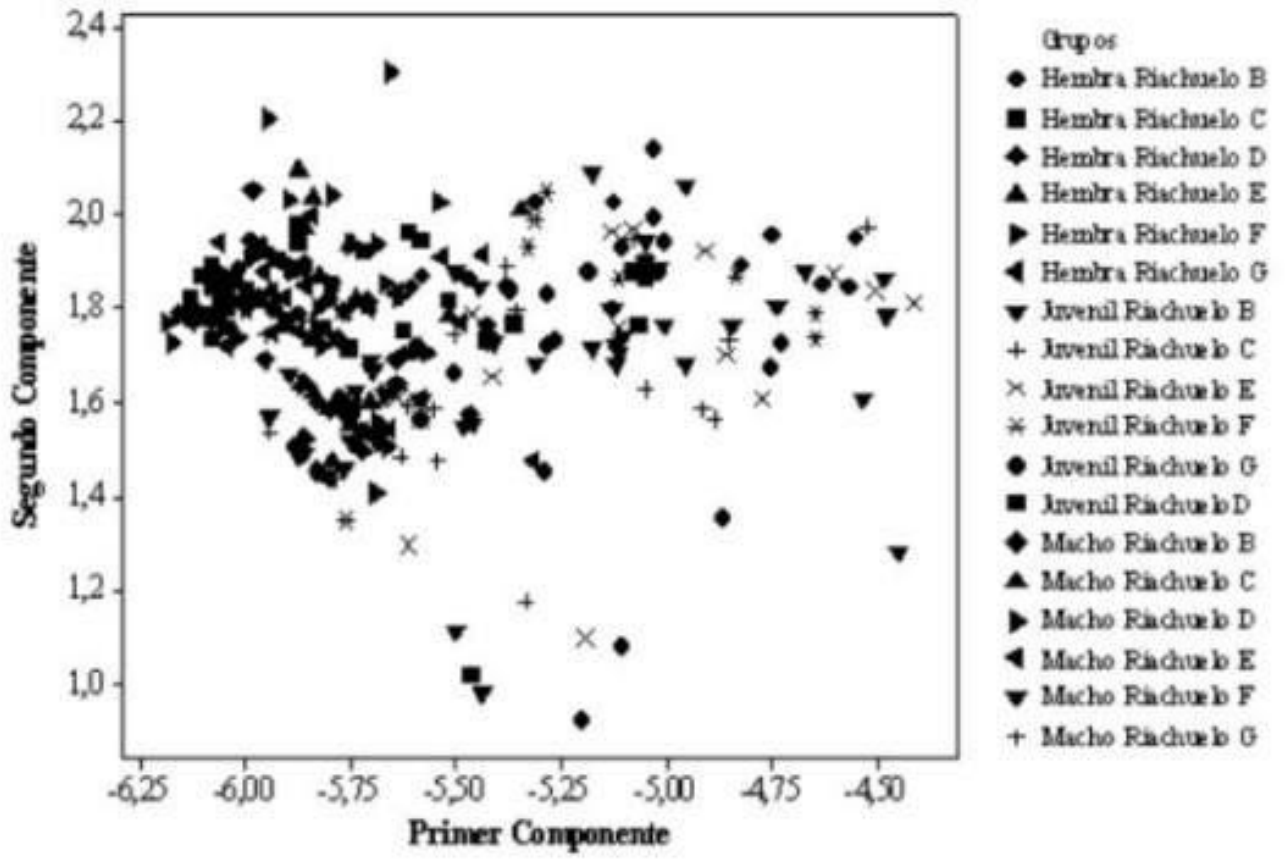

Figura 5. Diagrama de dispersión para los grupos de machos, hembras y juveniles por riachuelo en Isla Palma.

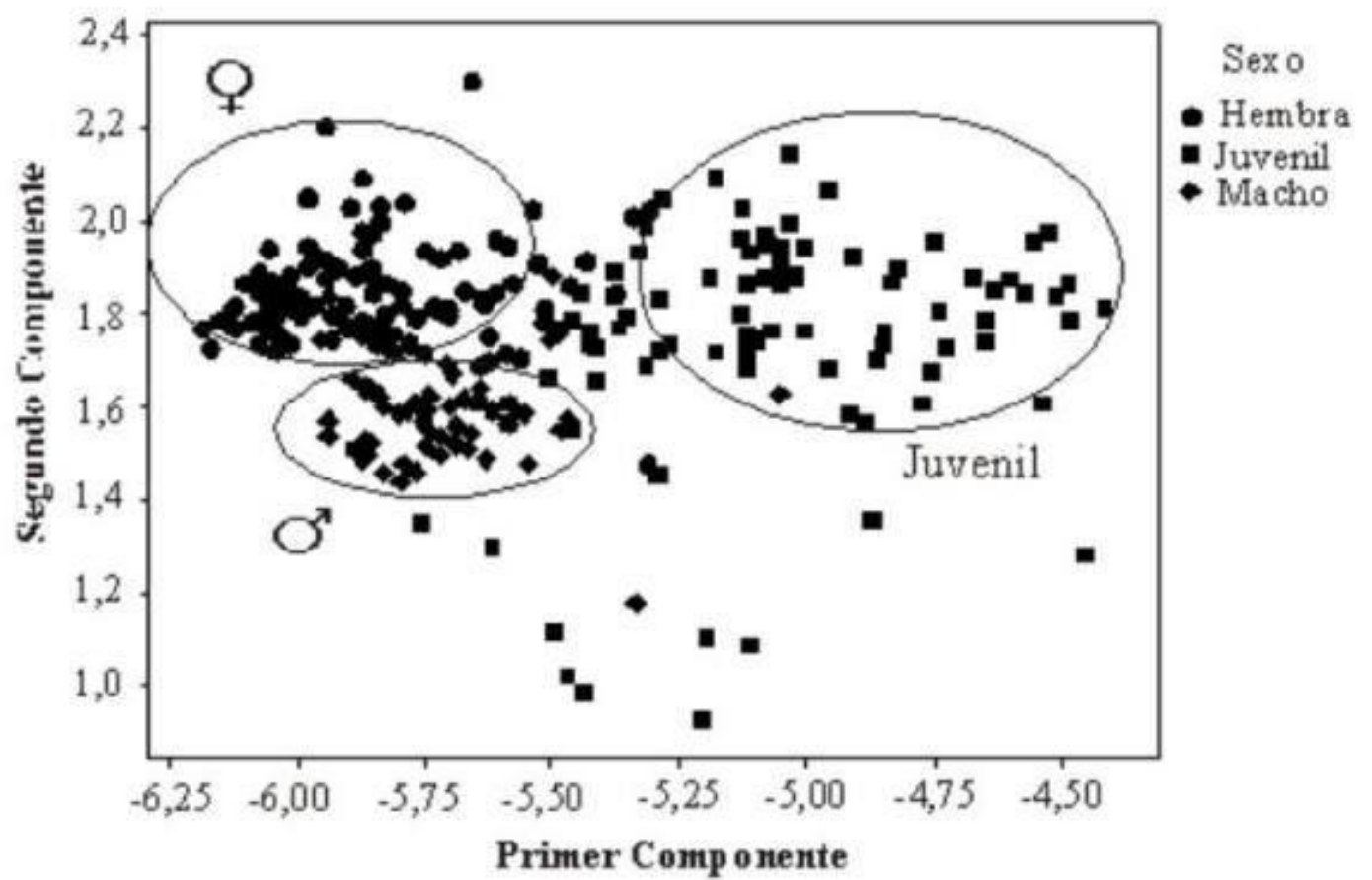

Figura 6. Diagrama de dispersión para los grupos de machos, hembras y juveniles por riachuelo en Isla Palma. 


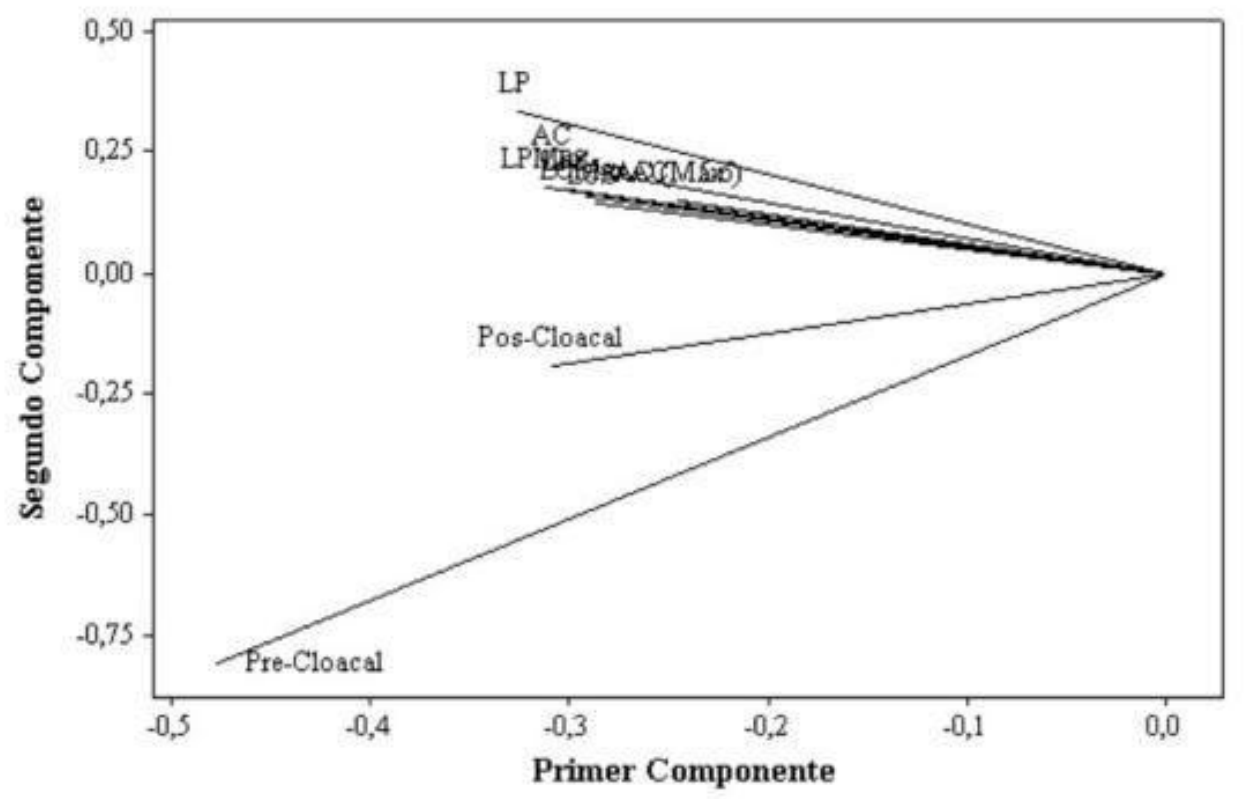

Figura 7. Grafica descriptiva del aporte de las variables corporales para los componentes uno y dos creados en el análisis de componentes principales.

\section{Discusión}

Los aspectos morfométricos relacionados con la especie $R$. nasuta no aparecen documentados ampliamente en la literatura, sin embargo hay algunos autores que han considerado el tema sugiriendo la existencia de dimorfismo sexual, en cuanto a talla y peso (MEDEM, 1962; LOAIZA, 2006; GARCÉS, 2008). Para el caso particular de la población de $R$. nasuta en Isla Palma la variación morfométrica y el dimorfismo sexual son concordantes con lo establecido por MEDEM (1962), siendo en términos generales las hembras significativamente más grandes que los machos. Por otra parte los juveniles no presentan un estado de diferenciación sexual visual por encontrarse en la fase de desarrollo y crecimiento. Esta variabilidad en la talla asociada al sexo probablemente esté fuertemente asociada con estrategias defensivas 0 de supervivencia de la especie. Por ejemplo, hembras más grandes y pesadas en comparación pueden albergar huevos más grandes y garantizar una mejor defensa en el momento en que sean más vulnerables como ocurre durante la puesta (GIBBONS y TINKLE, 1969). Por otro parte, una menor talla en los machos podría significar una menor inversión energética en crecimiento pero un incremento en capacidad de movilidad, condición pertinente para la población en estudio como lo sugiere PÉREZ (2007), además que esta condición incrementa la capacidad de localizar hembras, 
facilidad de cortejo e inseminación forzada (CHEN y LUE, 2001). En resumen, la diferenciación morfométrica entre machos y hembras de $R$. nasuta podría asociarse fuertemente con la teoría de selección sexual en tortugas propuesta por BERRY y SHINE (1980).

Una condición interesante de esta población, fue que el mismo patrón de variabilidad de talla se detectó en las poblaciones de los riachuelos estudiados, sin encontrarse variaciones significativas entre los individuos que conforman los diferentes grupos estudiados. Por lo tanto, la identificación de la longitud pre-cloacal como parámetro que más aporta a la variabilidad entre sexos adquiere una mayor relevancia; este resultado fue concordante con lo descrito por GARCÉS (2008) al evaluar una población de $R$. nasuta continental e insular en la Bahía de Málaga. La diferencia en la longitud precloacal entre los machos y las hembras de $R$. nasuta puede ser el resultado de diferencias fisiológicas asociadas al proceso de cópula en los machos y ovoposición en las hembras (MÁRQUEZ, 1995). Esta condición es más relevante en la especie estudiada debido a que se considera que ponen los huevos proporcionalmente más grandes del mundo (PRITCHARD y TREBBAU, 1984).

De acuerdo con GIRALDO et al. (2006), la diferenciación entre adultos y juveniles en $R$. nasuta se presenta a partir de $14 \mathrm{~cm}$ de longitud del caparazón. Sin embargo, de acuerdo con los resultados de este trabajo esta medida parece estar subestimando la capacidad reproductiva en esta especie, ya que se encontraron individuos de menor tamaño que presentaron una activa eversión de penes, lo que sugiere capacidad reproductiva. Por lo tanto, al momento de realizar un proceso de diferenciación etaria en $R$. nasuta se requiere no solo tener en cuenta la longitud total del caparazón, sino que es necesario considerar la longitud pre-cloacal y en lo posible confirmar la presencia de pene.

Frecuentemente se ha asociado el dimorfismo sexual en adultos a las condiciones ambientales locales y/o depredadores naturales (GIBBONS y LOVICH, 1990). En el caso de $R$. nasuta es poco probable que estos factores desempeñen un papel significativo en la variabilidad detectada, ya que Isla Palma presenta condiciones ambientales favorables y no está reportado en la isla depredadores naturales considerables tales como cocodrilos o caimanes, lo que probablemente favorece el desarrollo de un fenotipo homogéneo en la población estudiada.

\section{Agradecimientos}

A John Carr por el apoyo de traducción del resumen, Alan Giraldo por el apoyo comentarios y sugerencias en este manuscrito. A la Dirección Marítima Nacional de la 
República de Colombia - DIMAR por facilitar el acceso a Isla Palma para la realización de la fase de campo. Este trabajo fue financiado parcialmente por la Vicerrectoría de Investigaciones, la Facultad de Ciencias, el Departamento de Biología y el Grupo de Investigación en Ecología Animal de la Sección de Zoología de la Universidad del Valle, y recursos del Turtle Research Fund de la Fundacion de la Universidad de Louisiana.

\section{Referencias}

AMAYA, V. 2003. Relaciones ecológicas entre Echinometra vanbrunti y la macrofauna asociada a sus cavidades de habitación, en el acantilado verde de Isla Palma, Bahía de Malaga, Pacífico Colombiano. Tesis de pregrado, Universidad del Valle, Cali, Colombia.

ANÓNIMO. 2002. A Global Action Plan for Conservation of Tortoises and Freshwater Turtles. Turtle Conservation Fund.

ARESCO, M.; DOBIE, J. 2000. Variation in Shell Arching and Sexual Size Dimorphism of River Cooters, Pseudemys concinna from River Systems in Alabama. Journal of Herpetology 34:313-317.

BERRY, J. F.; SHINE, R. 1980. Sexual Size Dimorphism and Sexual Selection in Turtles (Order Testudines). Oecologia (Berl.) 44:185-191.

BOLKER, B. M.; HOLYOAK, M.; KRIVAN, V.; ROWE L.; SCHMITZ, O. 2003. Connecting theoretical and empirical studies of trait-mediated interactions. Ecology 84:1101-1114.

CAGLE, F. R. 1939. A system of marking turtles for future identification. Copeia 1939:170-173.

CANTERA, J. R.; NEIRA, R.; RICAURTE, R. 1998. Bioerosión en la costa pacífica colombiana. Un estudio sobre la biodiversidad, la ecología y el impacto humano de los animales destructores de los acantilados rocosos. Universidad del Valle, Departamento de Biología, Sección de Biología Marina. Fondo FEN Colombia. Colombia.

CASTELLANOS, G. A. 2003. Interacciones tróficas y espaciales de un ensamblaje de peces de charcos intermareales en un acantilado rocoso tropical, Bahía MalagaPacífico Colombiano. Tesis de pregrado, Universidad del Valle, Cali, Colombia.

CHEN, T. H.; LUE, Y. K. 2001. Growth Patterns of the Yellow-Margined Box Turtle (Cuora flavomarginata) in Northern Taiwan. Journal of Herpetology 36:201-208.

CORREDOR, G.; KATTAN, G.; GALVIZ, C. A.; MOROCHO, D. 2007. Tortugas del Valle del Cauca. Corporación Autónoma Regional del Valle del Cauca-CVC. Cali Colombia. 
DEPECKER, M.; BERGE, C.; PENIN, X.; RENOUS, S. 2006. Geometric morphometrics of the shoulder girdle in extant turtles (Chelonii). J. Anat 208:35-45.

GARCES, M. 2008. Estructura Poblacional, Variación Morfométrica y Dimorfismo Sexual de Rhinoclemmys nasuta (TESTUDINATA: EMYDIDAE) en dos Localidades del Pacifico Vallecaucano. Tesis de pregrado, Universidad del Valle, Cali, Colombia.

GIBBONS, J. W. 1990. Sex ratios and their significance among turtle populations. Pags 171 - 182 en: Gibbons, J. W. (ed.), Life History and Ecology of the Slider Turtle Smithsonian Inst. Press, Washington, DC.

GIBBONS, J. W.; LOVICH, J. E. 1990. Sexual dimorphism in turtles with emphasis on the slider turtle (Trachemys scripta). Herpetol. Monogr 4:1-29.

GIBBONS, J.W.; TINKLE, D. W. 1969. Reproductive variation between turtle populations in a single geographic area. Ecology 50:340-341.

GIRALDO, A.; CARR, J. L.; LOAIZA-NARANJO, J.; PEREZ-ARTEAGA, J. V.; ALEGRIA, J. L. 2006. Contribución al conocimiento de la biología y ecología de la tortuga hicotea blanca (Rhinoclemmys nasuta Boulenger 1902) en el Pacífico colombiano. Pags 114 115 en: Andrade-C, M.G., Aguirre C, J. y Rodríguez-Mahecha, J.V. (eds.), Segundo Congreso Colombiano de Zoología. Libro de Resúmenes Editorial Panamericana Formas e Impresos, Bogotá, Colombia.

KELLER, C. 1997. Ecología de poblaciones de Mauremys leprosa y Emys orbicularis en el Parque Nacional de Doñana. Tesis Doctoral, Universidad de Sevilla. Sevilla, España. LOAIZA, J. 2006. Aspectos Ecológicos de la tortuga Hicotea blanca (Rhinoclemmys nasuta Boulenger, 1902) en Isla palma, Bahía Málaga - Pacífico Colombiano. Tesis de pregrado, Universidad del Valle, Cali, Colombia.

MÁRQUEZ, C. 1995. Historia Natural y Dimorfismo Sexual de la Tortuga Kinosternon scorpioides en Palo Verde Costa Rica. Revista Ecología Latino Americana 2:37-44.

McCOY, M. W.; BOLKER, B. M.; OSENBERG, C. W.; MINER, B. G.; VONESH, J. R. 2006. Size correction: comparing morphological traits among populations and environments. Oecologia 148:547-554.

MEDEM, F. 1962. La distribución geográfica y ecológica de los Crocodylia y Testudinata en el departamento del Chocó Revista de la Academia Colombiana de Ciencias Exactas, Físicas y Naturales 11(44): 279-342.

MEDEM, F. 1976. Recomendaciones respecto a contar el escamado y tomar las dimensiones de nidos, huevos y ejemplares de los Crocodylia y Testudines. Revista. Lozania (acta zoológica Colombiana). 20 (1976):1-16. 
MERCHÁN, M. 2003. Contribución Al Conocimiento De La Biología De La Tortuga Negra (Rhinoclemmys funerea) Y La Tortuga Roja (R. pulcherrima manni) En Costa Rica. Tesis de Doctorado. Universidad Complutense De Madrid, Madrid España.

PÉREZ, J. V. 2007. Tasa de crecimiento y rango habitacional de Rhinoclemmys nasuta en Isla palma-Pacifico colombiano. Tesis de pregrado, Universidad del Valle, Cali Colombia.

PRITCHARD, P. C. H.; TREBBAU, P. 1984. Turtles of Venezuela. Soc Study Amph. Rept, Ohio.

ROMER, A. S. 1956. The Osteology of the Reptiles. University Chicago Press, Chicago.

ROWE, J. 1997. Growth Rate, Body Size, Sexual Dimorphism and Morphometric Variation in Four Populations of Painted Turtles (Chrysemys picta bellii) from Nebraska. American Midland Naturalist. 138:174-188.

RUEDA-ALMONACID, J.V.; CARR, J. L.; MITTERMEIER, R. A.; RODRIGUEZMAHECHA, J. V.; MAST, R. B.; VOGTH, R. C.; RHODING, A. G. J.; DE LA OSSAVELASQUEZ, J.; RUEDA, J. N.; MITTERMEIER, C. G. 2007. Las Tortugas y los Cocodrilianos de los Países Andinos del Trópico. Conservación Internacional, Bogotá, Colombia.

SARA, M.; FAVALORO, E.; MAZZOLA, A. 1999. Comparative morphometrics of sharpsnout seabream (Diplodus puntazzo Cefti, 1777), reared in different conditions. Aquaculture Research 19:195-209.

SOLER , J.; MARTÍNEZ- SILVESTRE, A. 2005. La tortuga Gigante Africana Geochelone (centrochelys) sulcata. Animalia 176:58-62.

TELLERÍA, J. L. 1991. Zoología Evolutiva de los Vertebrados. Editorial Síntesis, S.A., Madrid.

WERNER, E. E.; PEACOR, S. D. 2003. A review of trait-mediated indirect interactions in ecological communities. Ecology 84:1083-1100.

WEATHERS W. W.; WHITE F. N. 1971. Physiological thermoregulation in turtles. Am. J. Physiol 221:704-710. 